\title{
Differential expression of proteins related to START checkpoint of the cell cycle in human stomach, lung, cervix and liver cancers
}

\author{
Jae-Ryong Kim, ${ }^{1}$ Seong-Yong Kim, ${ }^{1}$ \\ Mi-Jin $\mathrm{Kim}^{2}$ and Jung-Hye $\mathrm{Kim}^{1,3}$ \\ 1 Department of Biochemistry College of Medicine, \\ Yeungnam University, Daegu, 705-035, Korea \\ 2 Department of Pathology, College of Medicine, \\ Yeungnam University, Daegu, 705-035, Korea \\ 3 Corresponding author \\ Accepted 2 June 1997
}

Abbreviations : PCNA, proliferating cell nuclear antigen; CDK4, cyclin dependent kinase 4; pRb, retinoblastoma protein; CDIs, cyclin dependent kinase inhibitors; NSCLC, nonsmall cell lung cancer; SCLC, small cell lung cancer; PCR, polymerase chain reaction; SSCP, single-strand conformation polymorphysm; CDKN2, cyclin dependent kinase inhibitor 2

\begin{abstract}
START (restriction) checkpoint in late G1 of the cell cycle is important in the regulation of cell proliferation. Many tumors have shown abnormalities partly in the components such as $p 53$, proliferating cell nuclear antigen (PCNA), retinoblastoma protein ( $p R b)$, cyclin dependent kinase 4 (CDK4), cyclin D1 and cyclin dependent kinase inhibitors (CDIs) were involved in the START checkpoint. To determine the differential expression of p53, PCNA, CDK4 and pRb in most common human cancers in Korea, immunohistochemical analyses for these proteins were performed on each 20 formalin-fixed and paraffin-embedded tumor tissues of stomach adenocarcinomas, lung cancers, cervix cancers and liver cancers. Significant differences existed in the expression of the proteins among the cancers from different anatomic sites. Stomach adenocarcinomas (55\%) and small cell lung cancers (SCLCs) $(70 \%)$ had high rate of p53 overexpression. Overexpression of $\mathrm{pRb}$ was shown in $70 \%$ of stomach adenocarcinomas and $90 \%$ non small cell lung cancers (NSCLSs). SCLCs had the highest rate $(\mathbf{8 0} \%)$ of $\mathbf{p R b}$ negativities. Cervix cancers showed the highest rate $(60 \%)$ of CDK4 overexpression and lower rate (15\%) of p53 overexpression. Liver cancers had the highest rate $(90 \%)$ of PCNA overexpression and the lowest rate $(10 \%)$ of p53 overexpression. Our results indicate that, at least, one of the abnormalities in p53, PCNA, CDK4 or pRb function occurs very commonly in these cancers,
\end{abstract}

thereby, suggesting that components in restriction checkpoint play a critical role in the development of these cancers through functional inactivation of $\mathrm{pRb}$.

Key words: START checkpoint, p53, PCNA, CDK4, $\mathrm{pRb}$

\section{Introduction}

Many human cancers are in part characterized by abnormalities in one or more of the genes responsible for regulating the cell cycle (Levine, 1993). The cell cycle is composed of G1, S, G2 and M phase. The transitions between different phases are regulated at checkpoints; such as restriction (START) checkpoint in G1 to $S, S$ phase checkpoint in $S$ to $G 2$ and mitotic checkpoint. These checkpoints are regulated by specific cyclins and cyclin-dependent kinases (CDKs). Especially, restriction checkpoint in late $\mathrm{G} 1$ is thought to be very important in control of the cell cycle. That is because, at this checkpoint, the cell commits itself to another round of DNA replication and many positive and negative regulators are integrated into the cell cycle (Hunter and Pines, 1994; Peter and Herskowitz, 1994). Many proteins such as cyclin D, CDK4 or CDK6, proliferating cell nuclear antigen (PCNA), cyclin-dependent kinase inhibitors (CDIs) and retinoblastoma protein $(\mathrm{pRb})$ are involved in the regulation of the restriction checkpoint. In late G1, cyclin D1 is bound to CDK4 and activates it. In addition to CDK4, cyclin D1 can form quaternary complexes with PCNA and p21. Cyclin D1-CDK4 complex can phosphorylate pRb, which is underphosphorylated and arrests cells in G1 phase. Phosphorylation of $\mathrm{pRb}$ relieves $\mathrm{G} 1$ arrest and allows cells to enter into S phase (Weinberg, 1995). Recently, CDIs such as p16, p15, p18 and p21 are found to be involved in the regulation of the restriction checkpoint. p16 inhibits CDK4 or CDK6 by binding in competition with cyclin D (Serrano et al., 1993). p15 binds to and inhibits specifically CDK4 in TGF $\beta$-treated cells (Hannon and Beach, 1994). p21 is a key mediator of p53-mediated growth suppression (El-Deiry et al., 1993) and high levels of p21 can inhibits the cyclin D-CDK4 complex (Zhang et al., 1994).

Some components in the restriction checkpoint are found to be implicated in the formation and progression of cancers through mutation or overexpression. An extremely high incidence of mutations and rearrangements of the p53 gene is observed in several human cancers, implicating it as the most significant tumor-suppressor gene to date (Hollstein et al., 1991). Aberrations of pRb, cyclin 
D1, p16 or PCNA are also found in many human cancers (Horowitz et al., 1990; Jang et al., 1993; Kamb et al., 1994; Nobori et al., 1994; Shapiro et al., 1995). All these abnormalities found in cancers at the restriction checkpoint are integrated finally into the loss or suppression of $\mathrm{pRb}$ function. Therefore, it is reasonable to infer that multiple disruptions of the growth-regulatory mechanisms defined by these proteins would be redundant ( $\mathrm{He}$ et al., 1995).

Stomach, lung, cervix and liver cancers are most common cancers in Korea. Various abnormalities in some components of restriction checkpoint have been partly observed in these cancers (Tamura et al., 1991; Jang et al., 1993; Hur and Hur, 1995; Wiethege et al., 1995; Kim et al., 1996). However, more systemic analysis may need to evaluate the important roles of these proteins in the formation and progression of cancers

In this study, we present differential expression of $p 53$, PCNA, CDK4 and pRb in paraffin-embedded tumor tissues of stomach, lung, cervix and liver cancers detected using immunohistochemical analysis.

\section{Materials and Methods}

\section{Tumor specimens}

Formalin-fixed and paraffin-embedded tumor tissues of 20 stomach adenocarcinomas, 20 lung cancers (10 NSCLCs and 10 SCLCs), 20 cervix cancers and 20 liver cancers, were obtained at Yeungnam University Hospital, Daegu, Korea.

\section{Materials}

Mouse monoclonal antibodies, anti-p53 (DO-1), anticyclinD1 (HD10) and anti-PCNA (PC10) and affinity-purified rabbit polyclonal antibodies, anti-p16 (M-156), anti-p21 (N-20), anti-pRb (C-15) and anti-CDK4 (C-22) antibody were purchased from Santa Cruz Biotechnology, Inc. (Santa Cruz, CA). Mouse monoclonal anti-human $\mathrm{pRb}$ (G3-245), anti-human cyclin D1 (G124-326) and polyclonal rabbit anti-human p16 antibody were obtained from
Pharmingen (San Diego, CA).

\section{Immunohistochemistry}

The immunohistochemical assay for detecting p53, PCNA, pRb, CDK4, cyclin D1, p21 and p16 in formalin-fixed and paraffin embedded tumor tissues was carried out using DAKO LSAB (DAKO, Carpinteria, CA) kit according to the instructions supplied by the manufacturer with minor modifications. Briefly, five- $\mu \mathrm{m}$ of paraffin sections of tumors dried on slide glasses treated with poly-L-lysine, were dewaxed twice in $100 \%$ xylene for $7 \mathrm{~min}$ and rehydrated through absolute, $95 \%$ and $70 \%$ ethanols. The sections were incubated in $3 \%$ hydrogen peroxide for 5 min and rinsed in $20 \mathrm{mM}$ Tris/Cl, $\mathrm{pH} 7.5,137 \mathrm{mM} \mathrm{NaCl}$ and $0.01 \%$ Tween 20 (TBS-T). Following boiling the sections in citrate buffer ( $\mathrm{pH} \mathrm{6.0)}$ for 5 min using a microwave oven, they were incubated with blocking solutions for $10 \mathrm{~min}$ and then reacted with antibodies at a 1:50 dilution in TBS-T at $4^{\circ} \mathrm{C}$ overnight. The incubation times with Link solution and Streptavidine peroxidase solution were lengthened to $30 \mathrm{~min}$. Chromogen (3\% 3amino-9-ethylcarbazole in N,N-dimethylformamide, AEC) was used for color development of all antibody stains. Hematoxylin was used as counterstain. Nuclear and cytoplasmic reactivity in tumor cells and nonneoplastic cells were separately graded as: absent, weak and strong.

\section{Results}

To examine the role of proteins involved in restriction checkpoint of the cell cycle in most common cancer in Korea, we carried out immunohistochemical ananlysis for p53, PCNA, CDK4, pRb, p21, cyclin D1 and p16 with 80 formalin-fixed and paraffin embedded tumor tissues of stomach adenocarcinomas, lung cancers, cervix cancers and liver cancers.

Figure 1 shows typical overexpression of $p 53$ in stomach adenocarcinoma (Figure $1 \mathrm{~A}$ ) and non-small cell lung cancer (Figure 1B). The strong immunoreactivity to
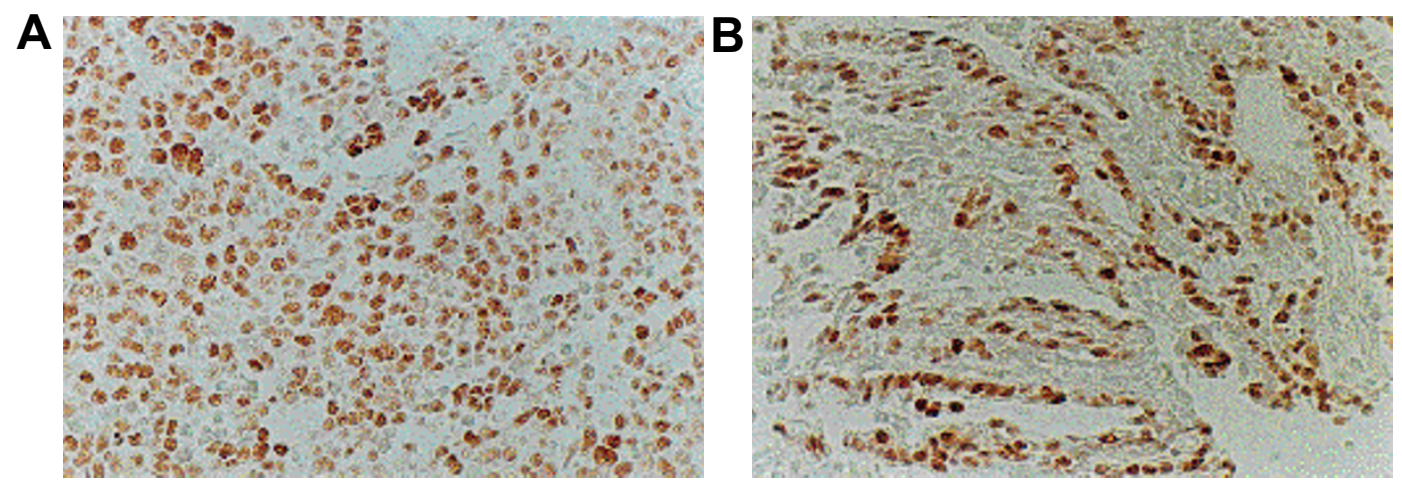

Figure 1. Typical p53 overexpression in stomach adenocarcinoma (A) and non-small cell lung cancer (B). The strong immunoreactivity to $\mathrm{p} 53$ is heterogenous and clearly localized to nuclei of tumor cells (200x). 

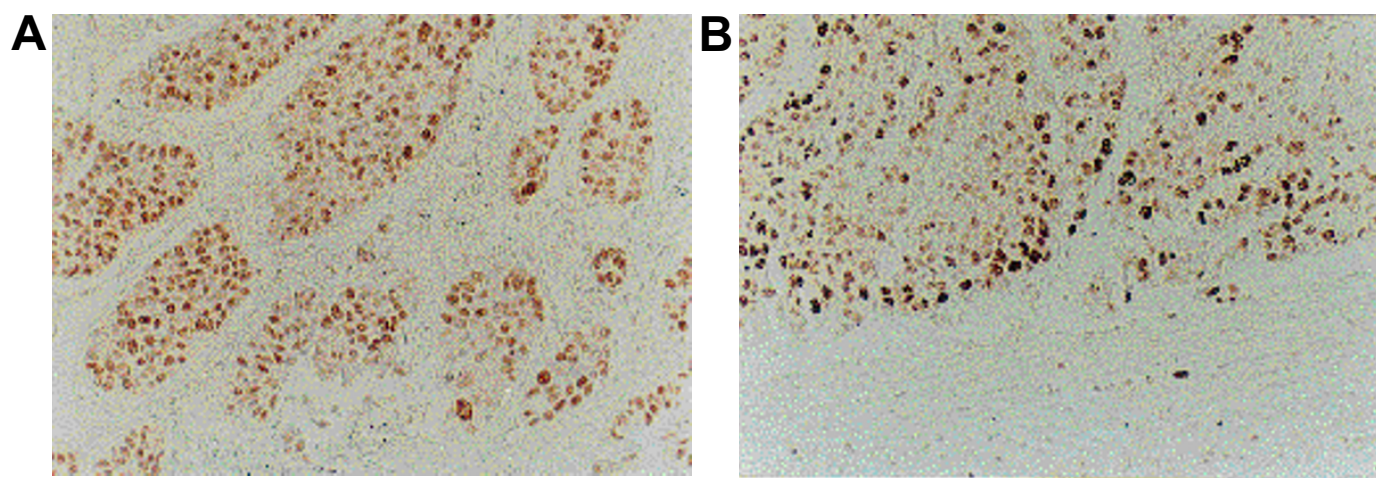

Figure 2. Typical PCNA overexpres-sion in non-small cell lung cancer (A) and liver cancer (B). Nuclear staining for PCNA is observed in tumor cells and admixed nonneo-plastic cells, whereas tumor cells show stronger reactivity to PCNA than nonneo-plastic cells (100x). p53 is heterogenous and clearly localized in nuclei of tumor cells.

Immunohistochemical analysis for $\mathrm{p} 53$ showed that 11 of the $20(55 \%)$ stomach adenocarcinomas, 10 of the $20(50 \%)$ lung cancers (in details, 3 of $10(30 \%)$ NSCLCs and 7 of $10(70 \%)$ SCLCs), 3 of the $20(15 \%)$ invasive cervix cancers and 2 of the $20(10 \%)$ liver cancers had clear nuclear staining only in the tumor cells, but not in nonneoplastic cells (Table 1 ).

In most of the 80 specimens exmained for PCNA, typical PCNA overexpression could be identified in nuclei both of tumor cells and nonneoplastic cells (Figure 2). However, nuclear reactivity to PCNA were different between tumor cells and admixed nonneoplastic cells. Overall, 10\% (2/20) in stomach adenocarcinomas, $70 \%(7 / 10)$ in NSCLCs,

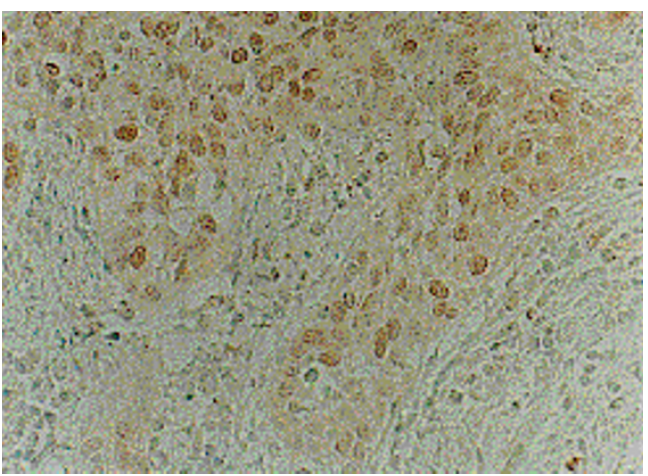

Figure 3. Typical CDK4 overexpression in cervix cancer. Tumor cells show strong nuclear and cytoplasmic staining and the reactivity is more intense in nuclei than in cytoplasm of tumor cells (200x).


Figure 4. Typical $\mathrm{pRb}$ overexpression in nonsmall cell lung cancer (A) and stomach adenocarcinoma (B). The immunoreactivity to $\mathrm{pRb}$ is stronger in tumor cells than in admixed nonneoplatic cells (200x).

Table 1. Differential overexpression of p53, PCNA, CDK4, and pRb in stomach, lung, cervix and liver cancers by immunohistochemistry

\begin{tabular}{lcccccc}
\hline & No. & $\begin{array}{c}\text { p53 } \\
\text { overexpression }\end{array}$ & $\begin{array}{c}\text { PCNA } \\
\text { overexpression }\end{array}$ & $\begin{array}{c}\text { CDK4 } \\
\text { overexpression }\end{array}$ & $\begin{array}{c}\text { pRb } \\
\text { overexpression }\end{array}$ & negativity \\
\hline $\begin{array}{l}\text { Stomach } \\
\text { adenocarcinoma }\end{array}$ & 20 & $11(55 \%)$ & $2(10 \%)$ & $3(15 \%)$ & $14(70 \%)$ & 0 \\
$\begin{array}{l}\text { Lung cancer } \\
\text { NSCLC }\end{array}$ & 10 & $3(30 \%)$ & $7(70 \%)$ & $3(30 \%)$ & $9(90 \%)$ & 0 \\
$\quad$ SCLC & 10 & $7(70 \%)$ & $3(30 \%)$ & $3(30 \%)$ & $1(10 \%)$ & $8(80 \%)$ \\
Cervix cancer & 20 & $3(15 \%)$ & $8(40 \%)$ & $12(60 \%)$ & $10(50 \%)$ & 0 \\
Liver cancer & 20 & $2(10 \%)$ & $18(90 \%)$ & $3(15 \%)$ & $6(30 \%)$ & $4(20 \%)$ \\
\hline
\end{tabular}


$30 \%(3 / 10)$ in SCLCs, $40 \%(8 / 20)$ in cervix cancers and $90 \%(18 / 20)$ in liver cancers showed more intense nuclear staining in tumor cells than in nonneoplastic cells in the same paraffin sections (Table 1).

In immunohistochemical staining for CDK4 (Figure 3), the nuclear and cytoplasmic reactivities were variable in 4 different tumor types. A tumor was considered to be CDK4 positive if there was nuclear staining in tumor cells, regardless of cytoplasmic staining. Fifteen percent (3/20) of stomach adenocarcinomas, $30 \%(6 / 20)$ of lung cancers, $60 \%(12 / 20)$ of cervix cancers and $15 \%(3 / 20)$ of liver cancers showed stronger nuclear CDK4 staining in tumor cells than in admixed nonneoplastic cells.

A tumor was considered to be $\mathrm{pRb}$ positive if there was nuclear staining in tumor cells and admixed nonneoplastic cells, regardless cytoplasmic staining, while $\mathrm{pRb}$ negative if there was no nuclear staining in tumor cells, whereas admixed nonneoplastic elements showed nuclear reactivity as a positive internal control. The nuclear reactivity for $\mathrm{pRb}$ was detected in all specimens. Typical $\mathrm{pRb}$ overexpression in stomach adenocarcinoma and NSCLC was shown in Figure 4. However, in 70\% (14/20) of stomach adenocarcinomas and 50\% (10/20) of cervix cancers, stronger nuclear staining was observed in tumor cells than in admixed nonneoplastic cells. Ninty percent (9/10) of NSCLCs showed the pRb overexpression, whereas $80 \%(8 / 10)$ of SCLCs were pRb negative. Twenty percent $(4 / 20)$ of liver cancers was identified to be $p R b$ negative.

Immunohistochemical analyses for p21, cyclin D1 and p16 were also performed in the 80 paraffin embeded tissues of 4 tumor types. However, we could not obtain any informative results.

Significant differences were observed in the expression of the proteins among the cancers from different anatomic sites (Table 1). Stomach adenocarcinomas had high rate of p53 (55\%) and pRb (70\%) overexpression and low rate of PCNA (10\%) and CDK4 (15\%) overexpression. Of interest, lung cancers showed clear differences in the expression of p53, PCNA and pRb between NSCLCs and SCLCs. NSCLCs showed a lower rate of p53 overexpression, a higher rate of PCNA overexpression and a higher rate in $\mathrm{pRb}$ overexpression than SCLCs, whereas SCLCs had a higher rate of p53 overexpression, a lower rate of PCNA overexpression and a higher rate in $\mathrm{pRb}$ negativities than NSCLCs.

Cervix cancers had the highest rate $(60 \%)$ of CDK4 overexpression and low rate (15\%) of p53 overexpression. Liver cancers had the highest rate $(90 \%)$ of PCNA overexpression and the lowest rate (10\%) of p53 overexpression.

Our results indicate that, at least, one of the abnormalities in p53, PCNA, CDK4 or pRb function occurs very commonly in tested four kinds of cancers, thereby, suggesting that components in restriction checkpoint play an critical role in the carcinogenesis and the progression of cancers.

\section{Discussion}

In immunohistochemical analysis for p53, the p53 overexpression was observed at a high rate in stomach adenocarcinomas (55\%) and SCLCs $(70 \%)$ and relatively at a low rate in NSCLCs (30\%), cervix cancers (15\%) and liver cancers $(10 \%)$. Clear nuclear staining to p53 was detected only in tumor cells but not in nonneoplastic cells. The abnormalities of p53 observed in various tumors are its mutations (deletions or missense mutations) or the loss of function due to rapid degradation by binding of the transforming proteins such as HPV E6, SV40 large $\mathrm{T}$ and adenovirus E1B protein. The p53 overexpression was frequently observed in many tumor cells, which is identified to have p53 missense mutations in exons 5 to 8. The missense mutations in $p 53$ were found to alter the conformation of protein and provide a longer half-life or greater stability in the cancer cells (Levine, 1993). Thus cancers with deletions, intronic mutations or missense mutations outside of exons 5 to 8 of p53 and cervix cancers infected with HPV 16 or 18 may show no nuclear staining in immunohistochemical analysis for p53. The p53 overexpression in stomach cancers were reported at the rate of $34 \%$ (Jang et al., 1993) and 58\% (Kim et al., 1996). Our results of $55 \%$ in stomach adenocarcinomas might be comparable to the previous reports. Jang et al. (1993) and Kim et al. (1996) pointed out that no significant differences in the p53 expression were found in terms of the tumor size, differentiations, tumor invasion or metastasis of stomach cancers. PCR-SSCP analysis of p53 mutation in primary stomach cancer (Tamura et al., 1991) showed that $64 \%$ of aneuploid tumors had mutations in exons 4-8. The rate of p53 overexpression in lung cancers was variable from $39-67 \%$ in NSCLCs (Ebina et al., 1994; Fontanini et al., 1994; Wiethege et al., 1995) and 35\% in SCLCs (Wiethege et al., 1995). In cervix cancers, the rate of p53 mutations was quite different between the results of either immunohistochemical $(42 \%)$ and PCR-SSCP (13\%) techniques (Kim and Kim, 1995). Our result in cervix cancers was comparable to that of PCR-SSCP analysis. Although we observed the lowest rate of p53 overexpression in liver cancers, the rate of $p 53$ mutations in these cancers showed a significant discordance between the findings of immunohistochemical and molecular techniques. Henkler et al. (1995) showed a very high frequency $(93 \%)$ of p53 mutation, as determined by using various antibodies and Hayashi et al. (1995) observed relatively low frequency $(28 \%)$ of mutation by PCR-SSCP.

PCNA is an auxiliary protein of DNA polymerases $\delta$ and $\varepsilon$, required for DNA replication and repair. PCNA is physically associated with all the known CDK-cyclin complexes (Ruddon, 1995). The PCNA overexpression 
was observed at the highest rate $(90 \%)$ in liver cancers. Hur and Hur (1995) also showed that the PCNA labeling index (percentage of positive cells per 100 tumor cells) in liver cancers was 10 times higher in tumor tissues than in nonneoplastic tissues and significantly increased as the tumor grade was higher. Maeda et al. (1996) also showed a higher PCNA labeling index in stomach cancers with lymph node metastasis than in those without lymph node metastasis. Kaneko and Izutsu (1995) showed a higher PCNA labeling index in cervix tumors of high grade than in those of low grade. In NSCLCs, PCNA overexpression was detected in the majority of tumor specimens compared to normal lung (Horowitz et al., 1990). The rate of PCNA positivity in NSCLCs was reported to be high as $72 \%$ (Wiethege et al., 1995) to $98 \%$ (Ebina et al., 1994), whereas SCLCs had a relatively low rate $(51 \%)$ of PCNA positivity (Wiethege et al., 1995).

CDK4 overexpression was found the highest rate in cervix cancers, which showed relatively low rate of p53 overexpression and PCNA overexpression. There was no reported about the aberrations of CDK4 in cervix cancers and other three cancers. Some investigators reported that CDK4 amplification is an alternative mechanism to CDKN2 homozyous deletion in glioma cell lines (Nobori et al., 1994) and glioma tissues (Sonoda et al., 1995). Therefore, further studies on CDK4 amplification in these cancers will be necessary.

$\mathrm{pRb}$ is a nuclear protein of 105 to $110 \mathrm{kDa}$ and its locus is located on chromosome $13 q 14$. Rb mutations or deletions has been seen at a high frequency in retinoblastoma, SCLCs, bladder and mammary carcinomas (Ruddon, 1995). The transforming proteins such as SV40 T, adenovirus E1A and HPV E7 were identified to affect carcinogenesis by binding to and inhibiting $\mathrm{pRb}$. In our results, SCLCs had the highest rate of $\mathrm{pRb}$ negativities, which is comparable to results from other reports (Hagashima et al., 1994; Geradts et al., 1995). The pRb overexpression observed in stomach adenocarcinomas, cervix cancers and NSCLCs might result from the increase of the phosphorylated $\mathrm{pRb}$. Our result of $20 \%$ of $\mathrm{pRb}$ negativities in liver cancer was the same as the results (20\%) reported by Hsia et al. (1994) and Zhang et al. (1994). Recently the differential inactivation of $p R b$ and CDKN2 were frequently identified in various tumors (Kelley et al., 1995; Ruddon, 1995).

We also carried out the immunohistochemical analyses for p21, cyclin D1 and p16 in the 80 paraffin embedded tissues of 4 tumor types. However, we could not obtain any informative results due to unresolved problems such as lack of the proper development of immunohistochemical methods for these proteins and differences in the specificity of commercially available antibodies.

Our results from PCR analysis for the $p 16$ gene (CDKN2) in the same tumor specimens showed that inactivations of CDKN2 due to mutations or hypermethylation were detected at a high frequency in these cancers.
The overexpression of cyclin D1 expression was identified frequently in primary NSCLCs (56\%) and NSCLC cell lines by Shapiro et al. (1995).

Lukas et al. (1995) suggested that cooperating aberrations within the cyclin D-CDK4-p16-pRb pathway are implicated in multistep oncogenesis and there are numerous candidate targets and potential oncogenic scenarios of deregulating the pathway. Four oncogenic scenarios have been documented as 1) loss of p16, 2) deregulated expression of cyclin D1, 3) cooperation of loss of p16 and overexpression of cyclin D1 and 4) lack of functional $\mathrm{pRb}$. Our results suggests that the very high frequency of one or more abnormalities in p53, PCNA, $\mathrm{CDK} 4$ or $\mathrm{pRb}$ among these cancers indicate that functional inactivation of $\mathrm{pRb}$ may play a critical role in the development of most cancers and, therefore, there might be additional oncogenic scenarios involved other than aberrations of CDK4, PCNA and p53.

\section{Acknowledgement}

This work was supported by a grant from the Ministry of Education for Basic Medical Science Research, Korea, 1995, awarded to Jung-Hye Kim and Jae-Ryong Kim.

\section{References}

Ebina, M., Steinberg, S. M., Mulshine, J. L. and Linnoila, R. I. (1994) Relationship of p53 overexpression and up-regulation of proliferating cell nuclear antigen with the clinical course of non-small cell lung cancer. Cancer Res. 54: 2469-2503

El-Deiry, W. S., Tokino, T., Velculescu, V. E., Levy, D. B., Parsons, R. Trent, J. M., Lin, D., Mercer, E., Kinzler, K. W. and Vogelstein, B. (1993) Waf1, a potential mediator of p53 tumor suppression. Cell 75: 817-825

Fontanini, G., Vignati, S., Bigini, D., Merlo, G. R., Ribecchini, A. Angeletti, C. A., Basolo, F., Pingitore, R. and Bebilacqua, C. (1994) Human non-small cell lung cancer: p53 protein accumulation is an early event and persists during metastatic progression. $\mathrm{J}$. Pathol. 174: 23-31

Geradts, J., Kratzke, R. A., Niehans, G. A. and Lincoln, C. E. (1995) Immunohistochemical detection of the cyclin-dependent kinase inhibitor $2 /$ multiple tumor suppressor gene 1 (CDKN2/MTS1) product $p 16^{I N K 4 A}$ in archival human solid tumors: correlation with retinoblastoma protein expression. Cancer Res. 55: 6006-6011

Hagashima, M., Doi, O., Kodama, K., Yokouchi, H. and Tateishi, R. (1994) Retinoblastoma protein expression in lung cancer: an immunohisto-chemical analysis. Oncology 51: 544-551

Hannon, G. J. and Beach, D. (1994) $p 15^{I N K 4 B}$ is a potential effector of TGF- $\beta$-induced cell cycle arrest. Nature 371: 257-261

Hayashi, H. sugi, K., Matsumata, T., Adachi, E. Takenaka, K. and Sugimachi, K. (1995) The clinical significance of p53 gene mutation in hepatocellular carcinomas from Japan. Hepatology 22: 1702-1707

He, J., Olson, J. J. and James, C. D. (1995) Lack of $p 16^{\text {INK4 }}$ or retino-blastoma protein $(\mathrm{pRb})$, or amplification-associated overexpression of cdk4 is observed in distinct subsets of malignant glial tumors and cell lines. Cancer Res. 55: 4833-4836

Henkler, F., Waseem, N., Golding, M. H., Alison, M.R. and Koshy, R. (1995) Mutant p53 but not hepatitis $B$ virus $X$ protein is present in hepatitis $B$ virus-related human hepatocellular carcinoma. Cancer Res. 55: 6084-6091 
Hollstein, M., Sidransky, D., Vogelstein, B. and Harris, C. C. (1991) p53 mutation in human cancers. Science 253: 49-53

Horowitz, J. M., Park, S. H., Bogenmann, E., Cheng, J. C., Yandell, D. W., Kaye, F. J., Minna, J. D., Dryja, T. P. and Weinberg, R. A. (1990) Frequent inactivation of the retinoblastoma antioncogene is restricted to a subset of human tumor cells. Proc.Natl. Acad. Sci. USA 87: 2775-2779

Hsia, C. C., Di Bisceglie, A. M., Kleiner, D. E., Farshid, M. and Tabor, E. (1994) RB tumor suppressor gene expression in hepatocellular carcinomas from patients infected with the hepatitis B virus. J. Med. Virol. 44: 67-73

Hunter, T. and Pines, J. (1994) Cyclins and cancer II: cyclin D and CDK inhibitors come of age. Cell $79: 573-582$

Hur, B. and Hur, M. H. (1995) Immunohistochemical investigation on expression of hepatitis $B$ surface antigen, transforming growth factor- $\alpha$, and proliferating cell nuclear antigen in hepatocellular carcinoma. Korean J. Pathol. 29: 478-491

Jang, W. I., Yang, W. I., Lee, C. I., Kim, H. S., Song, K. S., Cho, M. Y., Park, J. K. and Shim, Y. H. (1993) Immunohistochemical detection of p53 protein, c-erbB-2 protein, epidermal growth factor receptor protein and proliferating cell nuclear antigen in gastric carcinoma. J. Korean Med. Sci. 8: 293-304

Kamb, A., Gruis, N. A., Weaver-Feldhaus, J., Liu, Q., Harshman, K., Tavtigian, S. V., Stockert, E., Day III, R. S., Johnson, B. E. and Skolnick, M. H. (1994) A cell cycle regulator potentially involved in genesis of many tumor types. Science 264: 436-440

Kaneko, S. and Izutsu, T. (1995) Use of bromodeoxyuridine, proliferating cell nuclear antigen, and nucleolar organizer regions in the multiparametric assessment of proliferating potency in cervical intraepithelial neoplasia. J. Obstet. Gynaecol. 21: 133-144

Kelley, M. J., Nakagawa, K., Steinberg, S. M., Mulshine, J. L., Kamb, A. and Johnson, B. E. (1995) Differential inactivation of CDKN2 and Rb protein in non-small cell and small cell lung cancer cell line. J. Natl. Cancer Inst. 87: 756-761

Kim, D. H., Kim, Y. J., Yang, S. E., Paeng, S. S., Chang, H. J., Suh, J. I. and Park H. S. (1996) Immunohistochemical study of $\mathrm{p} 53$ and $\mathrm{nm} 23-\mathrm{H} 1$ protein in gastric carcinoma. Korean J. Pathol. 30: 587-594

Kim, K. H. and Kim, Y. S. (1995) Role of human papillomavirus and p53 tumor suppressor gene in cervical carcinogenesis. Yonsei Med. J. 36: 412-425 Levine, A. J. (1993) The tumor suppressor genes. Annu. Rev. Biochem. 62: 623-651

Lukas, J., Aagaard, L., Strauss, M. and Barteck, J. (1995) Oncogenic aberrations of p16 INK4/CDKN2 and cyclin D1 cooperate to deregulate G1 control. Cancer Res. 55: 48184823

Maeda, K., Chung, Y. S. Onoda, N., Ogawa, M., Kato, Y., Nitta, A., Arimoto, Y., Kondo, Y., Arakawa, T. and Sowa, M. (1996) Association of tumor cell proliferation with lymph node metastasis in early gastric cancer. Oncology 53: 1-5

Nobori, T., Miura, K., Wu, D. J., Lois, A., Takabayashi, K. and Carson, D. A. (1994) Deletions of the cyclin-dependent kinase-4 inhibitor gene in multiple human cancers. Nature 368: 753-756
Peter, M. and Herskowitz, I. (1994) Joining the complex: cyclin-dependent kinase inhibitory proteins and the cell cycle. Cell 79: 181-184

Ruddon, R. W. (1995) Cancer Biology, 3rd Edn. pp. 318-340, Oxford University Press

Serrano, M., Hannon, G. J. and Beach, D. (1993) A new regulatory motif in cell cycle control causing specific inhibition of cyclin D/CDK4. Nature 366: 704-707

Shapiro, G. I., Edwards, C. D., Kobzik, L., Godleski, J. Richards, W., Sugarbaker, D. J. and Rollins, B. J. (1995) Reciprocal Rb inactivation and $p 16^{\text {INK4 }}$ expression in primary lung cancers and cell lines. Cancer Res. 55: 505-509

Sonoda, Y., Yoshimoto, T. and Sekiya, T. (1995) Homozygous deletion of the MTS/p16 and MTS2/p15 genes and amplification of the CDK4 gene in glioma. Oncogene 11: 2145-2149

Tamura, G., Kihana, T., Nomura, K., Terada, M., Sugimura, T. and Hirohashi, S. (1991) Detection of frequent p53 gene mutations in primary gastric cancer by cell sorting and polymerase chain reaction single-strand conformation polymorphism analysis. Cancer Res. 51: 3056-3058

Weinberg, R. A. (1995) The retinoblastoma protein and cell cycle control. Cell 81: 323330

Wiethege, T., Voss, B. and Muller, K. M. (1995) p53 accumulation and proliferating-cell nuclear antigen expression in human lung cancer. J. Cancer Res. Clin. Oncol. 121: 371377

Zhang, H., Hannon, G. J. and Beach, D. (1994) p21-containing cyclin kinases exist in both active and inactive states. Genes Dev. 8: 1750-1758

Zhang, X., Xu, H. J., Murakami, Y., Sachse, R., Yashima, K., Hirohashi, S., Hu, S. X., Benedict, W. F. and Sekiya, T. (1994) Deletions of chromosome 13q, mutations in retinoblastoma 1 , and retinoblastoma protein state in human hepatocellular carcinoma. Cancer Res. 54: 4177-4182 